\title{
Isolated Fallopian Tube Torsion: Detorsion and Tubal Preservation
}

\author{
Shlomo M. Stemmer, MD, MS, Anna Shurshalina, MD, PhD \\ Department of Obstetrics and Gynecology, Thomas Jefferson University Hospital, Philadelphia, PA, USA (Dr. Stemmer). \\ Virtua Hospital, Voorhees, NJ, USA (Dr. Stemmer). \\ Genesis Biotechnology Group, Medical Diagnostic Laboratories LLC, Hamilton, NJ, USA (Dr. Shurshalina).
}

\begin{abstract}
Introduction: There has been a scarcity of cases of isolated fallopian tubes torsion in the literature.

Case Description: Isolated fallopian tube torsion in a 27 -year-old woman was associated with a hydrosalpinx. Laparoscopic detorsion of the right fallopian tube was performed within 24 hours of the onset of clinical symptoms. Surgical management was based on evaluation of tubal status and visual restoration of local perfusion as evidenced by the pink color of the untwisted tube and tested patency.

Discussion: Conservative surgical management to maximize fertility preservation should be the goal of treatment of women of reproductive age. Successful salvaging of tubal integrity rests on a low threshold for surgical management, the time from onset of symptoms to detorsion, the degree of tissue damage due to ischemia, and predisposing factors for tubal torsion.
\end{abstract}

Key Words: Tubal torsion, Hydrosalpinx, Laparoscopy.

Citation Stemmer SM, Shurshalina A. Isolated fallopian tube torsion: detorsion and tubal preservation. CRSLS e2015.00022. DOI: 10.4293/CRSLS.2015.00022.

Copyright (C) 2015 by SLS, Society of Laparoendoscopic Surgeons. This is an open-access article distributed under the terms of the Creative Commons Attribution-Noncommercial-ShareAlike 3.0 Unported license, which permits unrestricted noncommercial use, distribution, and reproduction in any medium, provided the original author and source are credited.

Address correspondence to: Anna Shurshalina, MD, PhD, Genesis Biotechnology Group, Medical Diagnostic Laboratories LLC, 2439 Kuser Rd, Hamilton, NJ 08690, USA. Telephone: (609) 570-1015, Fax: (609) 570-1030, E-mail: shurshalina@gmail.com

\section{INTRODUCTION}

Isolated fallopian tube torsion without ovarian involvement is a rarely reported event with an occurrence of 1 in 1.5 million women of reproductive age and is significantly less often encountered in the pediatric and adolescent age groups. ${ }^{1,2}$ The first clinical case of isolated tubal torsion was described in The Lancet in 1890 by Bland-Sutton. ${ }^{3}$ Several hundred reports have been cited in the literature since that time, predominantly in women of reproductive age and very rarely in premenarchal girls and postmenopausal women..$^{1,2,4-8}$ Most adnexal and tubal torsion cases are acute cases; however, there are 3 published cases of chronic tubal torsion: the first in a 34-year-old woman, the second in a 15-year-old adolescent without any identifiable risk factors, and the third in a 72 -year-old postmenopausal woman with ipsilateral hydrosalpinx. ${ }^{9-11}$ Isolated fallopian tube torsion also has been described in pregnancy, ${ }^{12,13}$ after surgical sterilization, ${ }^{14}$ in primary carci- noma of the fallopian tube, ${ }^{15}$ during labor, ${ }^{16}$ in tubal endometrioma, ${ }^{17}$ in polycystic ovarian syndrome, ${ }^{18}$ and in a fallopian tube remnant associated with the rudimentary horn in an adolescent girl with a unicornuate uterus. ${ }^{19}$

In this article we discuss diagnostic and treatment considerations for management of fallopian tube torsion and report the clinical case of a single twisted fallopian tube in a 27-year-old woman associated with hydrosalpinx that was successfully managed by laparoscopic detorsion.

\section{CASE DESCRIPTION}

A 27-year-old woman (gravida I, para 0, aborta I) was referred to our office by the emergency department, complaining of intermittent right lower-quadrant abdominal pain with gradual progression. She denied vaginal bleeding, fever, nausea, vomiting, dysuria, or changes in bowel habits. 


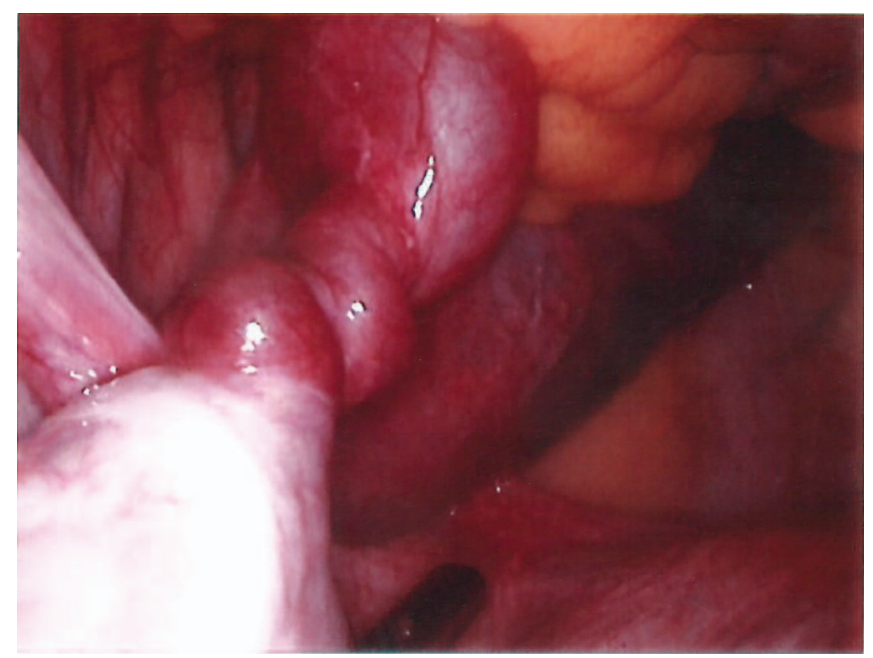

Figure 1. Laparoscopic findings: twisted right fallopian tube with a bluish tube color and hydrosalpinx.

The patient's medical history was unremarkable. The gynecologic history was significant for pelvic inflammatory disease and endometriosis treated with 3 laparoscopies and 3.75-mg monthly injections of leuprolide acetate for 6 months. The obstetric history was significant for 1 uncomplicated elective abortion; the patient was taking oral contraceptives. On physical examination, the vital signs were in normal range. The abdomen was tender mostly in the right lower quadrant, without rebound, guarding, or a palpable mass. Pelvic examination showed mild cervical motion tenderness, as well as right adnexal pain, and no adnexal mass was palpated. The complete blood count results, serum chemistry analysis findings, and sedimentation rate were all normal, and findings of a pregnancy test were negative. Transabdominal and transvaginal ultrasonography showed a 2 -cm simple cyst in the right ovary. There was no free air in the abdominal cavity or free fluid in the posterior cul-desac (pouch of Douglas).

The patient's intermittent, progressively worsening pain raised the possibility of adnexal torsion; therefore surgical intervention was recommended. She underwent laparoscopy, showing torsion of the right fallopian tube (Figure 1) accompanied by a large hydrosalpinx; a hydrosalpinx was also visible on the left tube. During laparoscopy, the end of the right fallopian tube was slightly bluish in its appearance, but after careful detorsion, it turned pink with a healthy-appearing color. The right ovary was not affected and was normal in appearance. Evaluation of the pelvis and abdomen showed normal ovaries and a normal appendix and large bowel. Examination of the cul-de-sac

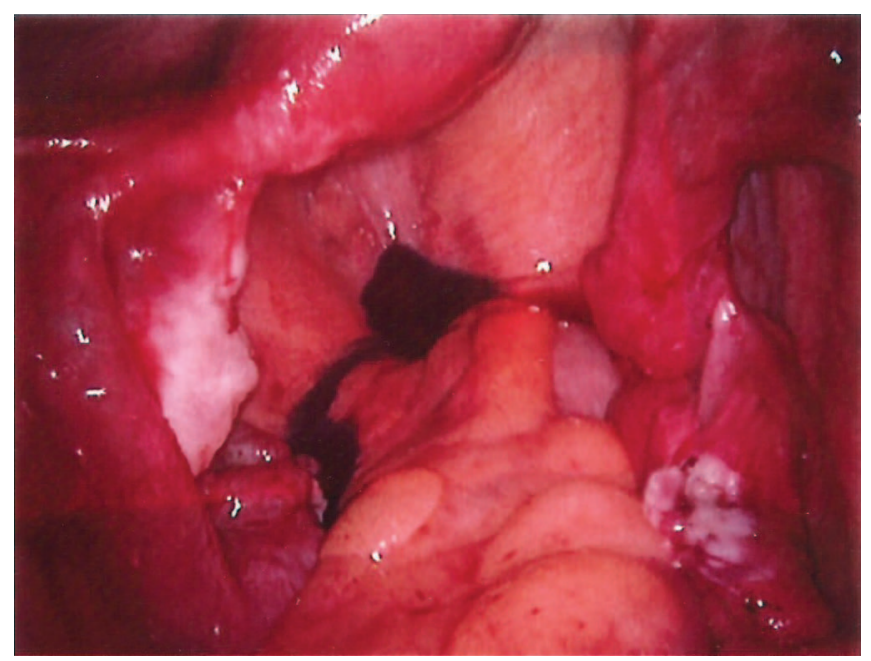

Figure 2. Laparoscopic findings: positive chromopertubation after tubal detorsion.

was notable for filmy adhesions in the area of the uterosacral ligaments bilaterally. Areas of endometriosis on both uterosacral ligaments were vaporized with a carbon dioxide laser. Bilateral drainage of the hydrosalpinx and fimbrioplasty were performed, followed by chromopertubation, which showed both tubes to be patent (Figure 2). The patient's postoperative recovery was uncomplicated, and she was discharged the same day.

\section{DISCUSSION}

The etiology of isolated torsion of the fallopian tubes is not well known. However, several risk factors have been identified, and these are divided into 2 groups: intrinsic and extrinsic ${ }^{2,4,20}$ (Table 1). On the basis of a literature review, Gaied et al ${ }^{1}$ concluded that primary (idiopathic) salpingeal torsion was diagnosed in $56 \%$ of cases and secondary torsion with underlying tubal pathology in $44 \%$. In our clinical case, the patient's history of pelvic inflammatory disease and the presence of endometriosis, pelvic adhesions, and bilateral hydrosalpinx can be identified as strong predisposing factors for torsion. The proposed mechanism for fallopian tube torsion begins with obstruction of the adnexal veins and lymphatic vessels, which leads to pelvic congestion and swelling inducing torsion. ${ }^{21}$

Isolated tubal torsion is usually unilateral and is 3 times more frequently diagnosed on the right side of the pelvis. The possible explanations for this asymmetry are the location of the sigmoid colon, which may preclude torsion of the left adnexa; a dextrorotated uterus; and the variance in venous return between the right and left fallopian 
Table 1.

Risk Factors Associated With Fallopian Tube Torsion

\begin{tabular}{ll}
\hline Intrinsic Tubal Risk Factors & Extrinsic Tubal Risk Factors \\
\hline Hydrosalpinx/hemosalpinx & $\begin{array}{l}\text { Ovarian/paraovarian or paratubal mass (especially benign mass } \\
\text { and mass } \geq 5 \mathrm{~cm} \text { in size) }\end{array}$ \\
Tubal ligation & $\begin{array}{l}\text { Adhesions (pelvic inflammatory disease, postsurgical, tuberculosis) } \\
\text { Endometriosis }\end{array}$ \\
Tubal neoplasms & Polycystic ovarian syndrome \\
Congenital tubal anomalies (eg, hydatid, cyst of Morgagni) & Pregnancy \\
Tubal hypermobility & Uterine tumors \\
Abnormal tubal peristalsis (drugs, autonomic dysfunction) & Pelvic congestion of mesosalpinx \\
& Trauma \\
& Sudden body position changes
\end{tabular}

tubes. ${ }^{22}$ However, rare cases of bilaterally occurring tubal torsion were reported in a 13-year-old premenarchal girl and a 36-year-old woman with infertility. ${ }^{23,24}$ Raziel et al25 discussed a case of recurrent isolated fallopian tube torsion 2 years after successful laparoscopic untwisting.

The absence of pathognomonic signs and symptoms, as well as nonspecific imaging findings and laboratory test results, makes tubal torsion challenging to diagnose. The diagnostic sensitivity of ultrasonography, computed tomography, and magnetic resonance imaging has been reported as 22\%, 14\%, and 40\%, respectively. ${ }^{1}$ Laparoscopy is considered the gold standard of diagnosis and treatment and is mandatory when torsion is suspected. The differential diagnosis for fallopian tube torsion includes acute appendicitis, ectopic pregnancy, pelvic inflammatory disease, torsed ovarian cyst, necrotic leiomyoma, and other urinary and gastrointestinal pathology.

In the clinical setting of suspected acute torsion, emergency surgical intervention is critical for diagnostic, therapeutic, and fertility-preservation purposes. Most of the risk factors for tubal torsion involve the pressure induced by the ovarian suspensory ligament or pelvic adhesions resulting in tubal circulatory events. Torsion of the fallopian tube around its vascular pedicle may lead to ischemia and cause necrosis depending on the remaining blood circulation and total time of severe ischemia. However, the exact length of torsion time that may lead to permanent tubal necrosis is not certain and can only be inferred from ovarian detorsion cases. Animal studies showed successful ovarian salvage after detorsion due to reperfusion if the time of torsion was $<36$ hours. ${ }^{26}$ In addition, rat models showed that gradual detorsion with a 5-minute pause can reduce reperfusion injury in ovarian torsion of 30 hours' duration. ${ }^{27}$

Conservative surgical management to maximize fertility preservation should be the goal of treatment of nulliparous young women and pregnant women. Salvage of potentially functional tubes, once cancer has been ruled out, should always be attempted. Successful tubal preservation depends on a low threshold for surgical management, time from onset of symptoms to detorsion, and degree of tissue damage due to ischemia. Early surgical intervention leads to salvage of the tube and fertility preservation; however, the correlation between the duration of clinical symptoms and rate of salpingectomy has not been described. ${ }^{28}$ The rate of conservative surgical treatment by tubal detorsion reported in the literature was about $12 \% .^{1}$

In our clinical case, we performed successful tubal detorsion within 24 hours of the onset of clinical symptoms. The surgical strategy was based on evaluation of the tubal status and visualization of the restoration of local perfusion as evidenced by the pink color of the untwisted tube and patency. The success of conservative surgical treatment in our case might be explained by the relatively slow progression of clinical symptoms, reversible stage of tubal ischemia, and surgical intervention performed in a timely manner.

\section{References:}

1. Gaied F, Emil S, Lo A, Baird R, Laberge JM. Laparoscopic treatment of isolated salpingeal torsion in children: case series 
and a 20-year review of the literature. J Laparoendosc Adv Surg Tech A. 2012;22:941-947.

2. Houry D. Abbott JT. Ovarian torsion: a fifteen-year review. Ann Emerg Med. 2001;38:156-159.

3. Bland-Sutton T. Salpingitis and some of its effects. Lancet. 1890;2:1146.

4. Van der Zanden M, Nap A, van Kints M. Isolated torsion of the fallopian tube: a case report and review of the literature. Eur J Pediatr. 2011;170:1329-1332.

5. Ozgun MT, Batukan C, Turkyilmaz C, Serin IS. Isolated torsion of fallopian tube in a post-menopausal patient: a case report. Maturitas. 2007;57:325-327.

6. Erikci VS, Hosgor M. Isolated salpingeal torsion in children: a case series and review of the literature. Ulus Travma Acil Cerrabi Derg. 2014;20:75-78.

7. Pampal A, Atac GK, Nazli ZS, Ozen IO, Sipahi T. A rare 182 cause of acute abdominal pain in adolescence: hydrosalpinx leading to isolated torsion of fallopian tube. I Pediatr Surg. 2012; 47:e31-e34

8. Comerci G, Colombo FM, Stefanetti M, Grazia G. Isolated fallopian tube torsion: a rare but important event for women of reproductive age. Fertil Steril. 2008;90:1198.e23-1198.e25.

9. Phillips K, Fino ME, Kump L, Berkeley A. Chronic isolated fallopian tube torsion. Fertil Steril. 2009;92:394.e1-394.e3.

10. Feng L, Liu Y, Liu H, Chen X. Chronic isolated fallopian tube torsion associated with huge hydrosalpinx and hemosalpinx in a postmenopausal woman: a case report and brief review. Eur J Obstet Gynecol Reprod Biol. 2012;164:235-236.

11. Schollmeyer T, Soyinka AS, Mabrouk M, Jonat W, Mettler L, Meinhold-Heerlein I. Chronic isolated torsion of the left fallopian tube: a diagnostic dilemma. Arch Gynecol Obstet. 2008;227:8790 .

12. Origoni M, Cavoretto P, Conti E, Ferrari A. Isolated tubal torsion in pregnancy. Eur J Obstet Gynecol Reprod Biol. 2009; 146:116-120.

13. Simsek Y, Ustun Y, Kaymak O, Akbaba E, Danisman N, Sirvan L. Hydronephrosis of pregnancy associated with torsion of the fallopian tube: a case report. Eur Rev Med Pharmacol Sci. 2011;15:448-451.

14. Sozen I, Kadako R, Fleischman S, Arici A. Diagnosis and laparoscopic management of a fallopian tube torsion following Irving tubal sterilization: a case report. Surg Endosc. 2002; $16: 217$.
15. Sanyal R, Chakraborty A, Ghosh D. Primary carcinoma of the fallopian tube with torsion. J Indian Med Assoc. 1989;87:145146.

16. Sorem KA, Bengtson JM, Walsh B. Isolated fallopian tube torsion presenting in labor. A case report. J Reprod Med. 1991; 36:763-764.

17. Wenger JM, Soave I, Lo Monte G, Petignat P, Marci R. Tubal endometrioma within a twisted fallopian tube: a clinically complex diagnosis. J Pediatr Adolesc Gynecol. 2013;26:e1-e4.

18. Tay J, Parker H, Dhange P, Paton-Forrester C, Atiomo W. Isolated torsion of the fallopian tube in a patient with polycystic ovarian syndrome (PCOS). Eur J Obstet Gynecol Reprod Biol. 2010;150:218-219.

19. Blitz MJ, Appelbaum H. Torsion of fallopian tube remnant associates with noncommunicating rudimentary horn in adolescent girl with unicornuate uterus. J Pediatr Adolesc Gynecol. 2014;27:e97-e99.

20. Youssef AF, Fayad MM, Shafeek MA. Torsion of the fallopian tube. A clinic-pathological study. Acta Obstet Gynecol Scand. 1962;41:292-309.

21. Bernardus RE, Van der Slikke JW, Roex AJ, Dijkhuizen GH, Stolk JG. Torsion of the fallopian tube: some considerations on its etiology. Obstet Gynecol. 1984;64:675-678.

22. Warner MA, Fleischer AC, Edell SL, et al. Uterine adnexal torsion: sonographic findings. Radiology. 1985;154:773-775.

23. Barisic D, Bagovic D. Bilateral tubal torsion treated by laparoscopy: a case report. Eur J Obstet Gynecol Reprod Biol. 1999; 86:99-100

24. Lima M, Libri M, Aquino A, Gobbi D. Bilateral hydrosalpinx with asynchronous tubal torsion: an exceptional finding in a premenarcheal girl. J Pediatr Surg. 2011;46:e27-e29.

25. Raziel A, Mordechai E, Friedler S, Schachter M, Pansky M, Ron-El R. Isolated recurrent torsion of the Fallopian tube: case report. Hum Reprod. 1999;14:3000-3001.

26. Anders JF, Powell EC. Urgency of evaluation and outcome of acute ovarian torsion in pediatric patients. Arch Pediatr Adolesc Med. 2005;159:532-535.

27. Ozkisacik S, Yazici M, Gursoy H, Culhaci N. Does gradual detorsion protect the ovary against ischemia-reperfusion injury in rats? Pediatr Surg Int. 2014;30:437-440.

28. Oltmann SC, Fischer A, Barber R, Huang R, Hicks B, Garcia N. Cannot exclude torsion-a 15-year review. J Pediatr Surg. 2009;44:1212-1216. 